\title{
The Spillover Effects of Supportive Neighboring Behavior on Mental Health and Career Satisfaction: A Longitudinal Research on Chinese Low-Income Employees
}

This article was published in the following Dove Press journal:

Psychology Research and Behavior Management

\section{Xiaoqian Zu' \\ Zhenduo Zhang' \\ Yongxiang $\mathrm{Wu}^{\prime}$ \\ Junwei Zheng ${ }^{2}$}

'School of Management, Harbin Institute of Technology, Harbin, People's Republic of China; ${ }^{2}$ Department of Construction Management, Kunming University of Science and Technology, Kunming, People's Republic of China
Correspondence: Xiaoqian Zu School of Management, Harbin Institute of Technology, No. 2, Yikuang Street, Nangang District, Harbin 15000I, People's Republic of China Email I5B910002@hit.edu.cn
Background: We examined the effects of supportive neighboring behavior on mental health and career satisfaction among Chinese low-income employees. We further examined the mediating roles of work interference with family (WIF) and of family interference with work (FIW) in this relationship.

Methods: A total of 220 Chinese low-income employees were selected via two-wave longitudinal survey in China; the time distance was five weeks. They completed questionnaires on their self-reported supportive neighboring behavior, work-family conflict, general mental health and career satisfaction. Afterwards, we adopted a structural equation modeling (SEM) to examine our hypotheses by R (Version 3.5.3) and Lavaan Package (Version 0.6-3). Results: Supportive neighboring behavior (Time 1) improved good mental health (Time 2) and career satisfaction (Time 2). Work interference with family (Time 1) mediated the effect of neighboring behavior on mental health while family interference with work (Time 1) mediated the effect of neighboring behavior on mental health and career satisfaction.

Conclusion: Our findings suggest that supportive neighboring behavior is vital in mitigating mental health problems and enhancing career satisfaction by decreasing work interference with family and family interference with work. Our research expands the scope of current literature on community support by incorporating bi-directional supportive neighboring behavior. By adopting family interference with work and work interference with family as mediators, our research examines the spillover mechanisms through which career satisfaction and mental health are influenced by supportive neighboring behavior.

Keywords: supportive neighboring behavior, mental health, career satisfaction, work interference with family, family interference with work

\section{Introduction}

Low-income employees are at high risk of developing mental health problems because of their low socioeconomic status. ${ }^{1}$ Due to the stress correlated with financial fragility, the status quo is that few low-income workers can simultaneously possess the high work-family benefits and sufficient social resources necessary to cope with these conflicts and pressures. ${ }^{2}$ Low-income employees have to rely on other resources to cope with mental health problems, and to achieve career success. ${ }^{3}$ Low-income employees have to rely on other support resources to cope with mental health problems, and career satisfaction, especially organizational and 
family support ${ }^{4,5}$ However, limited research explored the influences of community support on mental health and any spillover effects on career-related outcomes, especially for this special group of low income.

In building a harmonious community, neighboring behavior is a means for individuals to acquire social capital. Conventional research has been verified that received neighboring behavior, as a type of support, is part of social capital and constructively provides practical help to individuals. ${ }^{6,7}$ The given neighboring behavior (NBG)'s impact on conflicts is often positive and can serve as a means of resource acquisition. First, helping neighbors with their problems is a process of social exchange. From the perspective of social exchange, based on the grounds of reciprocity norms, the neighbors, as help receivers, help those who have helped themselves; thus, the given neighboring behavior also bolsters the social support that individuals receive. ${ }^{8}$ Second, helping others can promote positive emotions, such as self-confidence, a sense of accomplishment and a contented mood. In addition, from a learning perspective, when helping others, individuals can acquire cognitive processing skills, such as problemsolving. ${ }^{9}$ All these are part of individuals' social capital. ${ }^{10}$ As such, based on the literature, we treat given neighboring behavior and received neighboring behavior as an integrated concept and as a means through which individuals obtain social capital.

Community support is defined as assistance of various types exchanged between neighbors. ${ }^{3}$ According to ecological systems theory, social networks include work, family, and community microsystems, with resources from one system transferrable to the other two systems. ${ }^{11}$ Neighborhood frequently complements residents' network that exists in their residential environment. ${ }^{6}$ Neighbors' close spatial position makes them uniquely poised to help each other, both instrumentally and emotionally. ${ }^{12}$

It is a bidirectional rather than a unidirectional process to develop and maintain a social relationship. It yields an insufficient insight into the impacts of neighboring behavior on personal consequences to examine only one side of community support. ${ }^{13}$ Receiving helping from neighbors may supply low-income employees with external resources to deal with pressured events. ${ }^{3}$ Helping neighbors may enhance both cognitive and emotional regulation abilities in low-income employees, increasing their ability to cope with stress and to improve themselves, both in family and at work. ${ }^{14}$ Beyond the unidirectional received neighboring support, Perkins (1990) $)^{13}$ put forward the concept of neighboring behavior, referring to the extent to which residents take participation in their lived communities through giving and receiving assistance. In this vein, this study's first research question is to examine the influences of supportive neighboring behavior on mental health and career satisfaction.

Mental health refers to disorders that influence mood, thinking, and behavior (5rd ed., rev.; DSM-V-R ${ }^{15}$ ). Career satisfaction is defined as employees' assessment of their career progress, achievements, and anticipated outcomes, relative to their own goals and aspirations. ${ }^{16}$ Good mental health and career satisfaction have been regarded as desired outcomes in family and work domains. ${ }^{17}$ Thus, it is worth examining the spillover influences of supportive neighboring behavior on these two variables.

Furthermore, this study's second research question is to get deeper insights into mechanisms that link supportive neighboring behavior, mental health, and career satisfaction. In the past few years, the rise in dual-earner households has led to an increase in the number of employees who intertwine work with domestic roles. ${ }^{18}$ When stress from both work and family is mutually incompatible with each other, workfamily conflict arises, which conflicts between family and work roles. ${ }^{19}$ Work-family conflict is associated with damaged self-images in work and family domains, which are basic ingredients of an adult's identity, resulting in mental health decline and career dissatisfaction. ${ }^{20}$ In this vein, we examine family interference with work (FIW) and work interference with family (WIF), which are dimensions of work-family conflict, ${ }^{21}$ as mediators to evaluate how the resources from community microsystem spill over into family and work microsystems.

We adopted a two-wave longitudinal design to collect data from Chinese low-income employees to evaluate the conceptual model (see Figure 1). The current study was to explore the impacts of supportive neighboring behavior on mental health and career satisfaction. Further, we unveiled the mediating roles of family interference with work and work interference with family in the relationships among supportive neighboring behavior, career satisfaction and mental health. In doing so, our research provided two contributions to current research. First, our study introduces a unique approach by incorporating bidirectional supportive neighboring behavior as a critical antecedent to fostering good mental health and career satisfaction, beyond the effects of received neighboring behavior as explored in unidirectional studies. Second, this study uncovers the spillover mechanisms (work interference with family and family 


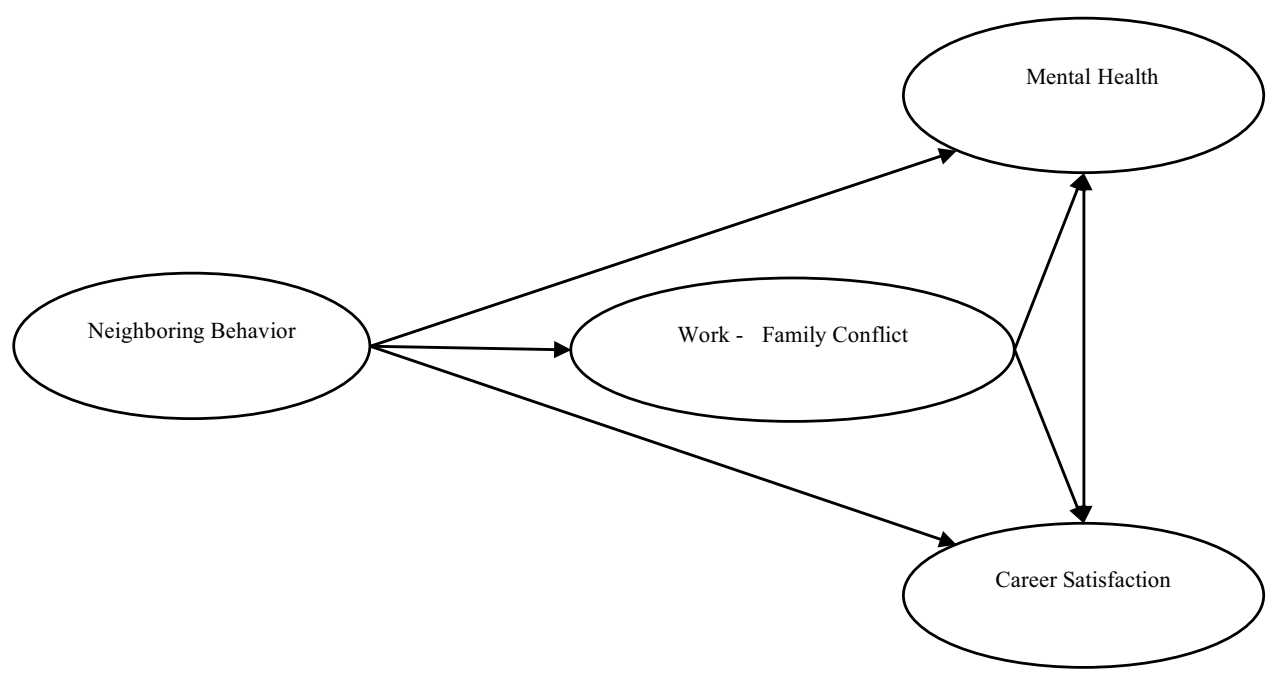

Figure I Conceputal model of relevance among neighboring behavior, work-family conflict, mental health and career satisfaction.

interference with work) through which supportive neighboring behavior influences family and work outcomes.

\section{Materials and Methods Data Collection and Samples}

We first developed a subject pool of 317 employee with incomes less than 28,000 Chinese yuan in 2018, an average yearly income for urban residents in Heilongjiang Province, China. All respondents were at least the age of 18 , with a regular work (40 hours per week) and they must have lived in their present communities in Heilongjiang Province for over one year.

Paper-based survey data collection occurred in two waves at the first week of November and the second week of December in 2018, respectively. We adopted demographic information, work-family conflict (including work interference with family and family interference with work) and supportive neighboring behavior in the first wave. Five weeks later, we collected mental health and career satisfaction in the second wave. The criterion for the validity of the questionnaire is that the participants have completely participated in the two surveys, and there is no obvious rule for the questionnaire. For example, answering a questionnaire showing a " $Z$ " shape change trend, or all items in the entire questionnaire are selected same option, will be rejected.

\section{Measures}

All the scales in our study were chosen from top peerreviewed English journals. A back-translation procedure was followed to translate them into Chinese. ${ }^{22}$
1. Supportive neighboring behavior. We used all ten items from Perkins et $\mathrm{al}^{13}$ to measure supportive neighboring behavior. This scale contained two dimensions: given neighboring behavior and received neighboring behavior. Sample items for each subdimension were "lend a hand to your neighbors when they are in emergency" and "receive advice from neighbors to solve a personal problem". In this study, we measure like the original scale, a five-point Likert scale was used with 1 indicating "low frequency" and 5 indicating "high frequency". This scale yielded a Cronbach's $\alpha$ score of 0.90 . Higher scores indicate more frequent supportive neighboring behavior.

2. Work-family conflict. We selected two distinct dimensions of work-family negative spillover and measured by an 8-item scale validated by Grzywacz and Marks, ${ }^{21}$ i.e., "family interference with work" (FIW) and "work interference with family" (WIF). Follow the original measurement method of the scale, using a five-point Likert scale $(1=$ never; $5=$ all the time). A sample item for family interference with work was "stress at home makes you irritable at work", and a sample item for work interference with family was "stress at work makes you irritable at home". The Cronbach's alpha score for family interference with work and work interference with family were both 0.96 for this scale. A higher score indicates a stronger conflict.

3. Mental health was measured using the original 12item general health questionnaire (GHQ) developed 
by Goldberg and Hiller ${ }^{23}$ and developed by Gao et $\mathrm{al}^{24}$ in Chinese samples. Sample items were "I have loss of sleep because of worrying" (depression and anxiety), "I am able to concentrate" (social dysfunction) and "I am usually thinking that I am self-worthless" (loss of confidence). GHQ was altered into a five-point Likert scale $(1=$ never; $5=$ all the times). Higher scores indicate worse mental health. The Cronbach's alpha for this scale was 0.93 .

4. Career satisfaction was selected a 3-item sub-scale developed by Martins et $\mathrm{al}^{25}$ using a five-point Likert scale as well as the original $(1=$ strongly disagree; $5=$ strongly agree). A sample item was "in general, I was satisfied with my career status". The Cronbach's alpha for this scale was 0.93. A higher score indicates greater career satisfaction.

\section{Analyses Methods}

Cronbach's alpha is used to measure the "sum" reliability of a group of synonymous or parallel measurement tools, and is a necessary tool for assessing the reliability of a questionnaire. ${ }^{26}$ Cronbach's Alpha is currently the most widely used reliability assessment tool in the development and use of scales in related fields such as psychology or education. The data in this research relates to the field of psychological and behavioral research, and participants samples are collected by using two-wave data collection methods. ${ }^{27}$ On this basis, this paper uses Cronbach's Alpha to measure the reliability of the questionnaire.

Common method variance (CMV) refers to the artificial covariation between predictor variables and criterion variables attributable to the same source or rater, the same measurement environment and project context. This artificial covariation severely confuses the research results and potentially misleads the conclusions, reducing the reliability of the data. However, in some research on organizational psychology, we observed that the 2-wave method can effectively reduce $\mathrm{CMV}^{28}$ Moreover, Chan et. al (and Wilkie et. al's study also confirmed that mental health is likely to change on a monthly basis. ${ }^{29,30}$ Given that other scholars have conducted causal inferences about mental health and other related variables based on the 2-wave method to varying degrees, this paper employed the 2-wave method to process data. ${ }^{31,32}$ Provided that the data was collected through self-reported questionnaire, we adopted confirmatory factor analysis (CFA) to test the possible influences of $\mathrm{CMV}$, using $\mathrm{R}$ (Version 3.5.3) and Lavaan Package (Version 0.6-3).
Supportive neighboring behavior and mental health both had multiple dimensions, we conducted the secondorder CFA to explore the second-order latent factors. For example, the items were loaded on "given neighboring behavior" and "received neighboring behavior" at first, then these two factors were loaded on the latent variable "supportive neighboring behavior". Furthermore, we adopted a structural equation modeling (SEM) to examine our hypotheses by R (Version 3.5.3) and Lavaan Package (Version 0.6-3). To test the indirect and direct effects, we used a bootstrapping method to generate the $95 \%$ confidence interval (CI) with 20,000 replications.

\section{Results}

In the end, 220 matched samples were eligible for analysis and the effective response rate was $69 \%$. Respondents worked in diverse industries in mainland China (e.g., the construction industry, manufacturing, and electronics). On average, respondents were 41.26 years old (standard deviation $=9.60) ; 41.4 \%$ were males $($ standard deviation $=0.49$ ), and $78.7 \%$ were married (standard deviation $=0.41$ ). As to education level, 19.1\% were senior school and below; $38.6 \%$ were high school; $22.7 \%$ were college; $15.9 \%$ were bachelor; and $3.7 \%$ were masters or above.

\section{Confirmatory Factor Analysis}

Results of confirmatory factor analysis indicated that the fivefactor expected model had an acceptable fit $\left(\chi^{2}(480)=1107.42\right.$, root-mean-square error of approximation $(\mathrm{RMSEA})=0.08$, root mean square residual $(\mathrm{RMR})=0.070$, comparative fit index $(\mathrm{CFI})=0.90)$.

\section{Descriptive Statistics}

Table 1 shows the results of mean, standard deviation and correlations among all studied variables. Supportive neighboring behavior was associated with decreased family interference with work $(r=-0.34, p<0.01)$, work interference with family $(r=-0.42, p<0.01)$, and poor mental health $(r=-0.51$, $p<0.01)$ and increased career satisfaction $(r=0.30, p<0.01)$. Family interference with work was associated with increased poor mental health $(r=0.41, p<0.01)$ and decreased career satisfaction $(r=-0.37, p<0.01)$. Work interference with family was associated with increased poor mental health $(r=0.41, p<0.01)$ and decreased career satisfaction $(r=-0.27, p<0.01)$. Poor mental health was associated with decreased career satisfaction $(r=-0.70, p<0.01)$.

Further, we conducted an independent $T$-test and one-way ANOVA test based on demographic variables, the results of 
Table I Mean, Standardized Deviance, and Correlations

\begin{tabular}{|c|c|c|c|c|c|c|c|c|c|}
\hline Variables & I & 2 & 3 & 4 & 5 & 6 & 7 & 8 & 9 \\
\hline \multicolumn{10}{|l|}{ I. Gender } \\
\hline 2. Age & 0.12 & & & & & & & & \\
\hline 3. Marital & 0.08 & $-0.39 * *$ & & & & & & & \\
\hline 4. Education & -0.08 & $-0.40 * *$ & $0.14 *$ & & & & & & \\
\hline 5. Neighboring behavior & 0.04 & -0.02 & $0.16^{*}$ & $0.24 * *$ & $(0.90)$ & & & & \\
\hline 6. FIW & 0.06 & $0.34 * *$ & -0.07 & $-0.52 * *$ & $-0.34 * *$ & $(0.96)$ & & & \\
\hline 7. WIF & 0.02 & $0.16 *$ & -0.04 & $-0.21 * *$ & $-0.42^{* *}$ & $0.33^{* *}$ & $(0.96)$ & & \\
\hline 8. Mental health & 0.06 & 0.11 & -0.06 & $-0.40 * *$ & $-0.5 I^{* *}$ & $0.4 I^{* *}$ & $0.4 I^{* *}$ & $(0.93)$ & \\
\hline 9. Career satisfaction & -0.12 & -0.07 & 0.08 & $0.22 * *$ & $0.30^{* *}$ & $-0.37 * *$ & $-0.27^{* *}$ & $-0.70 * *$ & $(0.93)$ \\
\hline Mean & 1.59 & 41.26 & 1.21 & 3.46 & 1.87 & 3.45 & 2.52 & 2.85 & 3.40 \\
\hline SD & 0.49 & 9.60 & $0.4 \mathrm{I}$ & 1.08 & 0.60 & 1.09 & 0.95 & 0.82 & 1.07 \\
\hline
\end{tabular}

Notes: Values in the parenthesis are Cronbach's alpha. $*_{p}<0.05 ; * *_{p}<0.01$.

Abbreviations: FIW, family interferece with work; WIF, work interference with family; SD, standard deviation.

which are shown in Table 2. Focal variables showed no significant differences between female and male. Married low-income employees had higher scores of supportive neighboring behaviors than single employees $(\mathrm{T}=-2.36$, $\mathrm{p}<0.05$ ). Higher education levels (bachelor above) correlated with higher supportive neighboring behavior $(\mathrm{F}=6.81$, $\mathrm{p}<0.01)$, better career satisfaction ( $\mathrm{F}=4.82, \mathrm{p}<0.01)$, lower mental health $(\mathrm{F}=13.53, \mathrm{p}<0.01)$ and lower work-family conflict $\left(\mathrm{F}_{\mathrm{FIW}}=37.48, \mathrm{p}<0.01 ; \mathrm{F}_{\mathrm{WIF}}=3.37, \mathrm{p}<0.01\right)$.

\section{Results of Structural Equation Modeling}

Results of structural equation modeling were shown in Figure 2 . We found that supportive neighboring behavior decreased family interference with work $(\beta=-0.32, p<0.01)$ and work interference with family $(\beta=-0.41, p<0.01)$. Family interference with work increased poor mental health $(\beta=0.20, p<0.01)$ and decreased career satisfaction $(\beta=-0.29, p<0.01)$. Work interference with family increased poor mental health $(\beta=0.17, p<0.01)$ and decreased career satisfaction $(\beta=-0.10, p<0.01)$. The direct relationships between supportive neighboring behavior, mental health $(\beta=-0.40, p<0.01)$, and career satisfaction $(\beta=0.23$, $p<0.01$ ) were significant.

Results in Table 3 showed that the direct effect of supportive neighboring behavior was significant, both for mental health (Effect $=-0.42,95 \% \mathrm{CI}=[-0.53,-0.32])$ and for career satisfaction (Effect $=0.28,95 \% \mathrm{CI}=[0.16,0.41])$. The indirect effects of supportive neighboring behavior on mental health were significant both through family interference with work $($ Effect $=-0.10,95 \% \mathrm{CI}=[-0.15,-0.05])$

Table 2 Demographic Characteristics of Variables

\begin{tabular}{|c|c|c|c|c|c|c|c|c|c|c|c|c|}
\hline & \multirow[t]{2}{*}{ Group } & \multirow[t]{2}{*}{$\mathbf{N}$} & \multicolumn{2}{|c|}{$\begin{array}{l}\text { Neighboring } \\
\text { Behavior }\end{array}$} & \multicolumn{2}{|c|}{ Mental Health } & \multicolumn{2}{|c|}{$\begin{array}{l}\text { Career } \\
\text { Satisfaction }\end{array}$} & \multicolumn{2}{|c|}{$\begin{array}{l}\text { Family } \\
\text { Interfere } \\
\text { Work }\end{array}$} & \multicolumn{2}{|c|}{$\begin{array}{l}\text { Work } \\
\text { Interfere } \\
\text { Family }\end{array}$} \\
\hline & & & Mean & $\mathbf{T} / \mathbf{F}$ & Mean & $\mathbf{T} / \mathbf{F}$ & Mean & $\mathbf{T} / \mathbf{F}$ & Mean & $\mathbf{T} / \mathbf{F}$ & Mean & $\mathbf{T} / \mathbf{F}$ \\
\hline \multirow[t]{2}{*}{ Gender } & Male & 91 & 1.85 & \multirow[t]{2}{*}{-0.53} & 2.79 & \multirow[t]{2}{*}{-0.93} & 3.55 & \multirow[t]{2}{*}{1.81} & 3.37 & \multirow[t]{2}{*}{-0.95} & 2.49 & \multirow[t]{2}{*}{-0.35} \\
\hline & Female & 129 & 1.89 & & 2.90 & & 3.29 & & 3.51 & & 2.54 & \\
\hline \multirow[t]{2}{*}{ Marital status } & Married & 173 & 1.82 & \multirow[t]{2}{*}{$-2.36^{*}$} & 2.88 & \multirow[t]{2}{*}{0.92} & 3.35 & \multirow[t]{2}{*}{-1.20} & 3.49 & \multirow[t]{2}{*}{0.98} & 2.54 & \multirow[t]{2}{*}{0.54} \\
\hline & Unmarried & 47 & 2.05 & & 2.76 & & 3.56 & & 3.31 & & 2.45 & \\
\hline Education & $\begin{array}{l}\text { Senior school and below } \\
\text { High school } \\
\text { College } \\
\text { Bachelor } \\
\text { Masters or above }\end{array}$ & $\begin{array}{l}42 \\
85 \\
50 \\
35 \\
8\end{array}$ & $\begin{array}{l}1.86 \\
1.73 \\
1.78 \\
2.20 \\
2.50\end{array}$ & $6.8 I^{* *}$ & $\begin{array}{l}3.06 \\
3.14 \\
2.79 \\
2.17 \\
2.11\end{array}$ & $13.53^{* *}$ & $\begin{array}{l}3.29 \\
3.18 \\
3.34 \\
4.05 \\
3.75\end{array}$ & $4.82 * *$ & $\begin{array}{l}3.76 \\
3.94 \\
3.52 \\
1.98 \\
2.63\end{array}$ & $37.48 * *$ & $\begin{array}{l}2.67 \\
2.65 \\
2.55 \\
2.04 \\
2.16\end{array}$ & $3.37 *$ \\
\hline
\end{tabular}

Notes: ${ }^{*} p<0.05 ; * * p<0.01$. 


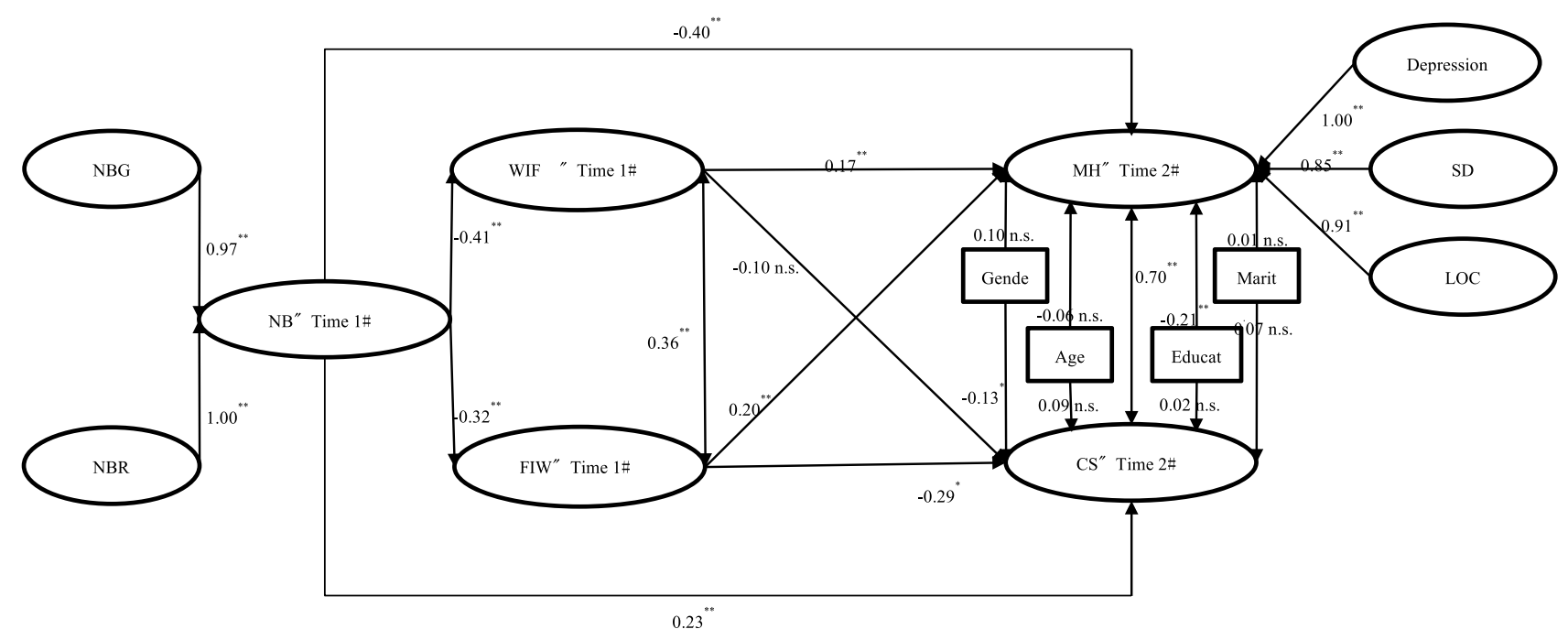

Figure 2 Conceputal model and results of relevance among neighboring behavior, work-family conflict, mental health and career satisfaction, with standardized beta weights. Notes: ${ }^{*} p<0.05$; ${ }^{* *} p<0.01$; Model fit: $\chi^{\prime \prime}(604)=1367.52$, the root mean square error of approximation $(\mathrm{RMSEA})=0.07$, the comparative fit index $(\mathrm{CFI})=0.90$, the Tucker-Lewis index $(\mathrm{TLI})=0.90$, the standardized root mean square residual $(\mathrm{SRMR})=0.07$.

Abbreviation: NBG, given neighboring behavior; NBR, received neighboring behavior; NB, neighboring behavior; WIF, work interference with family; FIW, family interferece with work; $\mathrm{MH}$, mental health; CS, career satisfaction; SD, social dysfunction; LOC, loss of confidence.

and work interference with family (Effect $=-0.05,95 \% \mathrm{CI}=$ $[-0.15,-0.05])$. The indirect effect of supportive neighboring behavior on career satisfaction was significant through family interference with work (Effect $=0.10,95 \% \mathrm{CI}=$ $[0.04,0.15])$.

\section{Discussion}

\section{Theoretical Implications}

The present study provided empirical evidence for the impacts of supportive neighboring behavior on mental health and career satisfaction. Previous research had mainly focused on the impact of received community support from neighbors on family and work outcomes (e.g., work-family conflict, job satisfaction and family performance $)^{3,33}$. This research extends this line of research by examining the influences of bi- directional, rather than uni-directional, supportive neighboring behavior since the establishment and maintenance of social relationships is an exchange process. ${ }^{34}$ Only with the transfer of mutual aid between neighbors can a reciprocal relationship develop. ${ }^{34}$ Previous research revealed that social relationships provide essential social resources for individuals to cope with stressful events ${ }^{35}$ and improve themselves. ${ }^{36}$ Given the scarce economic resources available to low-income employees, social resources are relatively important for them. ${ }^{3}$

In addition, we further explore how community-based social relationships influence low-income employees' family and work outcomes. Received support from neighbors helps low-income employees cope with potentially conflicting work-family demands. ${ }^{3}$ Giving support to neighbors helps low-income employees increase cognitive ability and

Table 3 Result of Bootstrapping Test

\begin{tabular}{|c|c|c|c|c|}
\hline Path & Effect & SE & 95\%LLCI & 95\%ULCI \\
\hline \multicolumn{5}{|l|}{ Indirect Effect } \\
\hline Neighboring Behavior $\rightarrow$ Work Interference with Family $\rightarrow$ Mental Health & -0.05 & 0.03 & -0.09 & -0.01 \\
\hline Neighboring Behavior $\rightarrow$ Family Interference with Work $\rightarrow$ Mental Health & -0.10 & 0.02 & -0.15 & -0.05 \\
\hline Neighboring Behavior $\rightarrow$ Family Interference with Work $\rightarrow$ Career Satisfaction & 0.10 & 0.03 & 0.04 & 0.15 \\
\hline \multicolumn{5}{|l|}{ Direct Effect } \\
\hline Neighboring Behavior $\rightarrow$ Mental Health & -0.42 & 0.05 & -0.53 & -0.32 \\
\hline Neighboring Behavior $\rightarrow$ Career Satisfaction & 0.28 & 0.06 & 0.16 & $0.4 I$ \\
\hline
\end{tabular}

Abbreviations: SE, standard error; LLCI, lower limit confidence interval; ULCI, upper limit confidence interval. 
emotional well-being. ${ }^{14}$ Moreover, both received and given neighboring behavior have long-lasting effects on social relationships between neighbors, providing low-income employees with ongoing social resources for maintaining good mental health ${ }^{37}$ and achieving career satisfaction in the workplace. ${ }^{35}$

By examining the mediation effect of both family interference with work and work interference with family, this research reveals the underlying mechanism through which career satisfaction and mental health are impacted by supportive neighboring behavior. Work-family conflict arises from incompatible stressors from work and family. For work interference with family, when employees are overly engaged in their work roles, they have reduced resources and time to fulfil their family roles. ${ }^{38}$ In this context, received neighboring behavior could directly offer low-income employees social resources to deal with family demands. ${ }^{3}$ Also, given neighboring behavior would enhance their cognitive information processing ability to solve other life problems, as well as enhancing their emotional regulation ability to better cope with family life. ${ }^{14}$ Cognitive information processing and emotional regulation are both critical to family roles and responsibilities, as well as to improve mental health.

Low-income employees have higher risks to experience domestic issues, ${ }^{39}$ leading to depletion of personal time and energy. Moreover, domestic issues spill over into work, resulting in maladaptive work behaviors, ${ }^{40}$ finally resulting in family interference with work. As in the above arguments, low-income employees might acquire support from social networks outside the work and family domains. ${ }^{41}$ For instance, women from low-income families rely heavily on neighbors for assistance with child-care and transportation, ${ }^{3}$ which may be associate with reduced family interference with work. On the other hand, given neighboring behavior is a process through which low-income employees help others solve problems, which in turn enhances cognitive information processing abilities and rewards them with positive emotions. ${ }^{42}$ In this vein, helping neighbors also helps low-income employees to acquire necessary resources to reduce influences of family interference with work, consequently improving their good mental health and career satisfaction. Bidirectional supportive neighboring behavior enhances low-income employees' social cohesion, which is examined as a buffer of work interference with family and family interference with work, further enhancing good mental health and career satisfaction.

\section{Practical Implications}

This study also provides several practical implications. This study held that supportive neighboring behavior could enable individuals to obtain social support from informal organizations and non-work sources, which would have a positive effect on individuals' psychological and behavioral formation to a certain extent. Neighborhood frequently complements residents' network that exists in their residential environment.

However, we posit that this behavior will not replace redistribution or reduce the social endeavor of low-income groups. This is because supportive neighboring behavior only provides individuals with a new path to obtain social capital, but this process may be indirect and slow. An individual's long-term social endeavor lays the foundations for shaping an individual's working conditions and living standards, where as the redistribution is the critical decision for the government to readjust factor-based income. These are long-term, irreplaceable means to adjust the income level of low-income groups.

Low-income employees should avoid social comparison and participate in social activities. They could develop beneficial personal social relationships through helping neighbors. Community managers should recruit skilled social workers, who can organize appealing and beneficial activities to improve social cohesion within a community. Overall, an amicable community climate motivates residents to interact with each other, facilitating recovery from stressful events.

\section{Conclusion}

This study of Chinese low-income employees attempted to explore the influences and underlying mechanisms of supportive neighboring behavior on mental health and career satisfaction. As expected in the conceptual model, supportive neighboring behavior has significant direct influences on mental health and career satisfaction. Furthermore, family interference with work mediates the indirect impacts of supportive neighboring behavior on mental health and career satisfaction. Work interference with family mediates the indirect influence of supportive neighboring behavior on mental health.

Our research has some limitations, guiding the direction for future research. First, we could not confirm causal relationships between our focal variables. Although a two-wave longitudinal design is beneficial for inferring causal effects of supportive neighboring behavior on mental health and career 
satisfaction, we cannot avoid the potential of a reversed causal relationship. ${ }^{33}$ Regarding the measurement method of the conversion scale, although we have adopted an adapted method, there may be slight measurement deviations. For future research, a multi-wave cross lagged analysis and a field experiment can be adopted to establish a firm causal effect of supportive neighboring behavior. Second, we could not rule out CMV. The results of CFA showed that CMV was not a serious problem in our research. However, it would be better to use multi-source data in future research. Third, our research is performed in a Chinese context. Supportive neighboring behavior, a core dimension of community social climate, varies between cultures. ${ }^{43}$ Future research may reexamine our research model in other cultures and other income classes, offering a cross-cultural understanding of the influences of supportive neighboring behavior.

\section{Approval of the Research Protocol}

All procedures performed in studies involving human participants were in accordance with the ethical standards of the Ethics Committee of Kunming University of Science and Technology and with the 1964 Helsinki declaration and its later amendments or comparable ethical standards.

\section{Consent for Publication}

Informed consent was signed and obtained from all individual participants included in the study.

\section{Informed Consent}

All study participants provided informed consent.

\section{Author Contributions}

All authors made substantial contributions to conception and design, acquisition of data, or analysis and interpretation of data; took part in drafting the article or revising it critically for important intellectual content; gave final approval of the version to be published; and agree to be accountable for all aspects of the work.

\section{Funding}

This study was supported by the National Natural Science Foundation of China (71701083).

\section{Disclosure}

The authors declare no conflicts of interest for this article.

\section{References}

1. Andrews AR, Jobe-Shields L, López CM, et al. Polyvictimization, income, and ethnic differences in trauma-related mental health during adolescence. Soc Psychiatry Psychiatr Epidemiol. 2015;50 (8):1223-1234. doi:10.1007/s00127-015-1077-3

2. Boushey H. Family-friendly policies: boosting mothers' wages. Cepr Rep Issue Briefs. 2005;5(3):341-344. doi:10.1080/09645299700000026

3. Griggs TL, Casper WJ, Eby LT. Work, family and community support as predictors of work-family conflict: a study of low-income workers. $J$ Vocat Behav. 2013;82(1):59-68. doi:10.1016/j. jvb.2012.11.006

4. Palmer JC, Komarraju M, Carter MZ, Karau SJ. Angel on one shoulder: can perceived organizational support moderate the relationship between the dark triad traits and counterproductive work behavior? Pers Individ Dif. 2017;110:31-37. doi:10.1016/j. paid.2017.01.018

5. Russo M, Shteigman A, Carmeli A. Workplace and family support and work-life balance: implications for individual psychological availability and energy at work. $J$ Posit Psychol. 2016;11 (2):173-188. doi:10.1080/17439760.2015.1025424

6. Jiang S, Li C, Wang Z, et al. The mediating effect of neighbor relationship between community social support and elderly's subjective well-being in China. J Health Psychol. 2017. doi:10.1177/ 135910531773353

7. Diamond-Smith N, Sudhinaraset M, Melo J, et al. The relationship between women|"s experiences of mistreatment at facilities during childbirth, types of support received and person providing the support in Lucknow, India. Midwifery. 2016;40:114-123. doi:10.1016/j. midw.2016.06.014

8. Bartlett MY, DeSteno D. Gratitude and prosocial behavior: helping when it costs you. Psychol Sci. 2006;17(4):319-325. doi:10.1111/ j.1467-9280.2006.01705.x

9. Grant AM. Leading with meaning: beneficiary contact, prosocial impact, and the performance effects of transformational leadership. Acad Manage J. 2012;55:458-476. doi:10.5465/amj.2010.0588

10. Bolino MC, Grant AM. The bright side of being prosocial at work, and the dark side, too: a review and agenda for research on other-oriented motives, behavior, and impact in organizations. Acad Manag Ann. 2016;10(1):599-670. doi:10.1080/19416520.2016.11 53260

11. Voydanoff P. The effects of community demands, resources, and strategies on the nature and consequences of the work-family interface: an agenda for future research. Fam Relat. 2005;54(5):583-595. doi:10.1111/j.1741-3729.2005.00343.x

12. Unger DG, Wandersman A. Neighboring in an urban environment. Am J Community Psychol. 1982;10(5):493-509. doi:10.1007/ BF00894140

13. Perkins DD, Florin P, Rich RC, Wandersman A, Chavis DM. Participation and the social and physical environment of residential blocks: crime and community context. Am J Community Psychol. 1990;18(1):83-115. doi:10.1007/BF00922690

14. Bolino MC, Grant AM. The bright side of being prosocial at work, and the dark side, too: a review and agenda for research on other-oriented motives, behavior, and impact in organizations. ANNALS. 2016;10(1):599-670. doi:10.5465/19416520.2016.1153260

15. American Psychiatric Association. Diagnostic and Statistical Manual of Mental Disorders $\left(D S M-5^{\circledR}\right)$. 5th ed. Washington, DC: American Psychiatric Publication; 2013.

16. Seibert SE, Kraimer ML. The five-factor model of personality and career success. J Vocat Behav. 2001;58(1):1-21. doi:10.1006/ jvbe. 2000.1757

17. Hirschi A, Herrmann A, Nagy N, Spurk D. All in the name of work? Nonwork orientations as predictors of salary, career satisfaction, and life satisfaction. $J$ Vocat Behav. 2016;95-96:45-57. doi:10.1016/j. jvb.2016.07.006 
18. Matias M, Ferreira T, Vieira J, Cadima J, Leal T, Mena Matos P. Workplace family support, parental satisfaction, and work-family conflict: individual and crossover effects among dual-earner couples. Appl Psychol. 2017;66(4):628-652. doi:10.1111/apps.12103

19. Greenhaus JH, Beutell NJ. Sources of conflict between work and family roles. AMR. 1985;10(1):76-88. doi:10.5465/amr.1985. 4277352

20. Ladge JJ, Little LM. When expectations become reality: work-family image management and identity adaptation. Academy of Management Review. 2019;44(1):126-149. doi:10.5465/amr.2016.0438

21. Grzywacz JG, Marks NF. Reconceptualizing the work-family interface: an ecological perspective on the correlates of positive and negative spillover between work and family. $J$ Occup Health Psychol. 2000;5(1):111-126. doi:10.1037/1076-8998.5.1.111

22. Brislin RW. Orientation programs for cross-cultural preparation. In: Marsella AJ, Tharp G, Giborowski TJ, editors. Perspectives in CrossCultural Psychology. Orlando, FL: Academic Press; 1979:87-304.

23. Goldberg DP, Hillier VF. A scaled version of the general health questionnaire. Psychol Med. 1979;9(1):139-145. doi:10.1017/ S0033291700021644

24. Gao F, Luo N, Thumboo J, Fones C, Li S-C, Cheung Y-B. Does the 12-item general health questionnaire contain multiple factors and do we need them? Health Qual Life Outcomes. 2004;2:63. doi:10.1186/ 1477-7525-2-63

25. Martins LL, Eddleston KA, Veiga JF. Moderators of the relationship between work-family conflict and career satisfaction. AMJ. 2002;45 (2):399-409. doi:10.5465/3069354

26. Tavakol M, Dennick R. Making sense of cronbach's alpha. Int J Med Educ. 2011;2:53-55. doi:10.5116/ijme.4dfb.8dfd

27. Killian KD. Development of the Vicarious Resilience Scale (VRS): a measure of positive effects of working with trauma survivors. Psychol Trauma. 2018;9:1. doi:10.1037/tra0000199

28. C M B, Khoury C. Organizational powerlessness, dehumanization, and gendered effects of procedural justice. J Manag Psychol. 2016;31 (2):570-585. doi:10.1108/JMP-09-2014-0267

29. Chan HY, Ho CM, Mahendran R, et al. Effects of horticultural therapy on elderly' health: protocol of a randomized controlled trial. BMC Geriatr. 2017;17:1. doi:10.1186/s12877-017-0588-z

30. Wilkie DP, Cicero DC, Mueller CW. Disruptive behavior treatment progress as a function of therapy focus and youth diagnosis in a community mental health setting. Adm Policy Ment Health. 2017;45(1):1-11.

31. Liang J, Farh CIC, Farh J-L. Psychological antecedents of promotive and prohibitive voice: a two-wave examination. Acad Manage J. 2012;55(1):71-92. doi:10.5465/amj.2010.0176
32. Costantini A, Ceschi A, Sartori R. A cognitive perspective on counterproductive work behavior. Evidence from a two-wave longitudinal study. Curr Psychol. 2019. doi:10.1007/s12144-019-00416-5

33. Li C, Jiang S, Li N, Zhang Q. Influence of social participation on life satisfaction and depression among Chinese elderly: social support as a mediator. J Community Psychol. 2018;46(3):345-355. doi:10.1002/ jcop. 21944

34. Cropanzano R, Mitchell MS. Social exchange theory: an interdisciplinary review. J Manage. 2005;31(6):874-900. doi:10.1177/ 0149206305279602

35. Park SG, Kang HJA, Lee HR, Kim SJ. The effects of LMX on gender discrimination and subjective career success. Asia Pac J Hum Resour. 2017;55(1):127-148. doi:10.1111/1744-7941.12098

36. Sconfienza C, Lindfors P, Lantz Friedrich A, Sverke M. Social support at work and mental distress: a three-wave study of normal, reversed, and reciprocal relationships. J Occup Health. 2019;61 (1):91-100. doi:10.1002/1348-9585.12020

37. Werner-Seidler A, Afzali MH, Chapman C, Sunderland M, Slade T. The relationship between social support networks and depression in the 2007 national survey of mental health and well-being. Soc Psychiatry Psychiatr Epidemiol. 2017;52(12):1463-1473. doi:10. 1007/s00127-017-1440-7

38. Halbesleben JRB, Harvey J, Bolino MC. Too engaged? A conservation of resources view of the relationship between work engagement and work interference with family. $J$ Appl Psychol. 2009;94(6):1452-1465. doi:10.1037/a0017595

39. Williamson HC, Altman N, Hsueh J, Bradbury TN. Effects of relationship education on couple communication and satisfaction: a randomized controlled trial with low-income couples. J Consult Clin Psychol. 2016;84(2):156-166. doi:10.1037/ccp0000056

40. Mercado BK, Dilchert S. Family interference with work and its relationship with organizational citizenship and counterproductive work behaviors. Int J Sel Assess. 2017;25(4):406-415. doi:10.1111/ ijsa. 12195

41. Lambert SJ. Lower-wage workers and the new realities of work and family. Ann Am Acad Pol Soc Sci. 1999;562(1):174-190. doi:10. 1177/000271629956200112

42. Lin KJ, Ilies R, Pluut H, Pan S-Y. You are a helpful co-worker, but do you support your spouse? A resource-based work-family model of helping and support provision. Organ Behav Hum Decis Process. 2017;138:45-58. doi:10.1016/j.obhdp.2016.12.004

43. Parks MJ. Who is our neighbor? Toward a multilevel and cross-national roadmap for building community capacity. J Community Pract. 2015;23(2):182-202. doi:10.1080/10705422. 2015.1027459
Psychology Research and Behavior Management

\section{Publish your work in this journal}

Psychology Research and Behavior Management is an international, peer-reviewed, open access journal focusing on the science of psychology and its application in behavior management to develop improved outcomes in the clinical, educational, sports and business arenas. Specific topics covered in the journal include: Neuroscience, memory and decision making; Behavior modification and management; Clinica applications; Business and sports performance management; Social and developmental studies; Animal studies. The manuscript management system is completely online and includes a very quick and fair peer-review system, which is all easy to use. Visit http://www. dovepress.com/testimonials.php to read real quotes from published authors. 